\title{
HYPOTHESIS: TETRACYCLINES FOR SEVERE COVID-19 - THE PERFECT REPURPOSING CANDIDATE?
}

\author{
Jose Garrido-Mesa ${ }^{1}$, Kate Adams², Julio Galvez ${ }^{3}$, and Natividad Garrido Mesa ${ }^{2}$ \\ ${ }^{1}$ King's College London Faculty of Life Sciences and Medicine \\ ${ }^{2}$ School of Health, Sport and Bioscience. University of East Lodon. E15 4LZ. \\ ${ }^{3}$ University of Granada
}

September 24, 2021

\begin{abstract}
We would like to draw attention to the potential of immunomodulatory tetracyclines for severe COVID-19. The COVID-19 pandemic is having a devastating impact on developing countries. A successful approach to manage the scarcity of cost-effective therapies worldwide is drug repurposing. Predictions of direct anti-viral activity of tetracyclines against SARS-CoV2 have been confirmed experimentally. Furthermore, their effectiveness in experimental ARDS has been proven extensively, counteracting the overt inflammatory reaction and fibrosis sequelae due to a synergic combination of pharmacological activities. Finally, a few clinical reports have confirmed their potentiall in COVID-19 patients, encouraging the development of this novel indication. We believe that the benefits of their multi-target pharmacology and their safety profile place immunomodulatory tetracyclines as gold repurposing candidates for COVID-19.
\end{abstract}

\section{HYPOTHESIS: TETRACYCLINES FOR SEVERE COVID-19 - THE PERFECT REPURPOSING CANDIDATE?}

Garrido-Mesa $\mathrm{J}^{* 1}$, Adams $\mathrm{KE}^{2}$, Galvez J ${ }^{3}$, Garrido-Mesa $\mathrm{N}^{4}$

${ }^{1}$ Department of Medical and Molecular Genetics, Faculty of Life Sciences and Medicine, Guy's \& St Thomas' NHS Foundation Trust and King's College London NIHR Biomedical Research Centre, London, UK.

${ }^{2}$ Department of Bioscience, School of Health, Sport and Bioscience, University of East London, London, UK.

${ }^{3}$ Department of Pharmacology, Center for Biomedical Research (CIBM), University of Granada, AND Instituto de Investigación Biosanitaria de Granada (ibs.GRANADA), Granada, Spain.

${ }^{4}$ Department of Pharmacy, School of Life Sciences, Pharmacy and Chemistry. Kingston University, London, UK.

*Correspondence : Jose Garrido Mesa, $\mathrm{PhD}$

Spondyloarthritis Research Group, Department of Medical \& Molecular Genetics, School of Basic and Medical Biosciences, Faculty of Life Sciences \& Medicine, King's College London. Email: j.garrido@kcl.ac.uk (J. Garrido-Mesa).

\section{Contributions:}

- GMJ - Conceptualization, Literature Search, Visualization, Writing - Original Draft Preparation 
- AKE - Literature Search, Writing - Original Draft Preparation

- GJ - Project Administration, Supervision, Review.

- GMN - Project Administration, Supervision, Writing - Review \& Editing

Conflict of interest: The authors declare that they have no known competing financial interests or personal relationships that could have appeared to influence the work reported in this paper

Keywords: Tetracycline, COVID-19, ARDS, Lung, Immunomodulatory, Repurposing

\section{ABSTRACT}

We would like to draw attention to the potential of immunomodulatory tetracyclines for severe COVID-19. The COVID-19 pandemic is having a devastating impact on developing countries. A successful approach to manage the scarcity of cost-effective therapies worldwide is drug repurposing. Predictions of direct anti-viral activity of tetracyclines against SARS-CoV2 have been confirmed experimentally. Furthermore, their effectiveness in experimental ARDS has been proven extensively, counteracting the overt inflammatory reaction and fibrosis sequelae due to a synergic combination of pharmacological activities. Finally, a few clinical reports have confirmed their potentiall in COVID-19 patients, encouraging the development of this novel indication. We believe that the benefits of their multi-target pharmacology and their safety profile place immunomodulatory tetracyclines as gold repurposing candidates for COVID-19.

\section{MANUSCRIPT}

The COVID-19 pandemic has positioned vaccines at the top of the therapeutic arsenal. "Born" to help us fight infections, their effectiveness has been demonstrated in the context of a multitude of infectious diseases, and there is no doubt of their key role in our current fight against COVID-19. However, even more remarkable is their adaptation and development to face new therapeutic challenges, such as fighting cancer. This is a strikingly similar story to that of another versatile therapeutic family, the tetracyclines. Following the discovery of their non-antibiotic properties, such as inhibition of matrix metalloproteinases (MMPs) and different immunomodulatory effects, they have been tested in many non-infectious conditions with promising results. However, their categorization as "antibiotic drugs" is holding back their clinical application to other pathological conditions, despite their proven potential and the development of a novel class of chemically modified tetracyclines (CMTs) devoided of antibiotic properties. Whilst CMTs could have a chance in a patent-dominated market, the pharmaceutical industry is not likely to get on board with drug repurposing strategies involving old tetracycline antibiotics, such as minocycline and doxycycline. Yet, the exceptional circumstances that characterise the current pandemic, particularly challenging for developing countries, demand cost-effective therapies that can be readily applied worldwide. Therefore, based on their well-described benefit in acute respiratory distress syndrome (ARDS) in general, and given recent reports of their activity against SARS-CoV2 infection, we would like to draw attention to and encourage the repurposing of tetracyclines to fight COVID-19.

As of end of July 2021, COVID-19 incidence worldwide remains very high. Despite the success of vaccination programs in developed countries, infections are rising again with the spread of new variants, whose impact on developing countries is far more devastating. The cumulative number of cases now exceeds 190 million and the number of global reported deaths exceeds 4 million. Severe COVID-19 cases require hospital care due to viral pneumonia progressing into ARDS, causing difficulty in breathing and low blood oxygen levels (Figure 1). However, in addition to direct respiratory failure (accounting for $70 \%$ of fatal COVID-19 cases), some may succumb to secondary bacterial and fungal infections. Furthermore, an aggressive inflammatory response (the 'cytokine storm') is strongly implicated in airway and multi-organ damage, leading to permanent sequelae or death by septic shock and circulatory failure.

Why do we believe that immunomodulatory tetracyclines could help facing this pathological setting? Many of their pharmacological activities have already proven useful in ARDS and viral infections. Starting with 
their antibiotic properties, tetracyclines are effective and particularly useful in pneumonias and infections caused by hospital-adquired multi-resistant bacteria. Hence, rather than a limitation, their antibiotic activity would play an important role against secondary bacterial pneumonia in COVID-19 patients (Figure 1 A).

\section{Anti-viral activity}

Strikingly, several reports have revealed promising antiviral effects of tetracyclines in infections caused by RNA viruses (Figure 1 B), such as HIV, Dengue virus, Japanese encephalitis virus and others. It has been suggested that tetracyclines could interact and stabilize dsRNA (Dutta and Basu, 2011), which are involved in viral replication and activate host defense mechanisms, as observed with minocycline in HIV infection (Szeto et al., 2010). Regarding SARS-CoV2, several of its functions are associated with the host MMPs, and may be susceptible to tetracyclines' MMP inhibitory activity. Bioinformatic analysis to prioritise drug repurposing candidates based on reported activities and molecular docking with SARS-CoV2 proteins have predicted potential anti-viral activity for several tetracycline analogs (Wu et al., 2020). Docking studies propose binding of doxycycline to the human Adaptor-Associated Kinase 1 (AAK1) and the viral ADPribose phosphatase (ADPRP) (Sayed et al., 2020), involved in viral endocytosis and replication, respectively. It has also been proposed that tetracycline and doxycycline could act as inhibitors of ACE2-spike binding (Sachdeva et al., 2020; Zhao and Patankar, 2021), and doxycycline and minocycline as inhibitors of the SARS-CoV-2 main protease ( $\mathrm{M}^{\text {pro }}$ ) (Bharadwaj et al., 2020). In fact, experimental evidence of tetracycline's direct antiviral effect against SARS-CoV2 has already been reported. Doxycycline has shown to reduce viral entry and replication in Vero E6 cells infected with SARS-CoV-2 with a EC $_{50}$ of $4.5+-2.9 \mu \mathrm{M}$ (Gendrot et al., 2020), which is compatible with the bioavailability of current formulations. Furthermore, tetracyclines could also attain antiviral activity indirectly. Viruses exploit the mitochondria machinery and aerobic glycolysis of infected cells, which could also be susceptible to the impact of tetracyclines on mitochondrial dynamics, mainly due to calcium buffering.

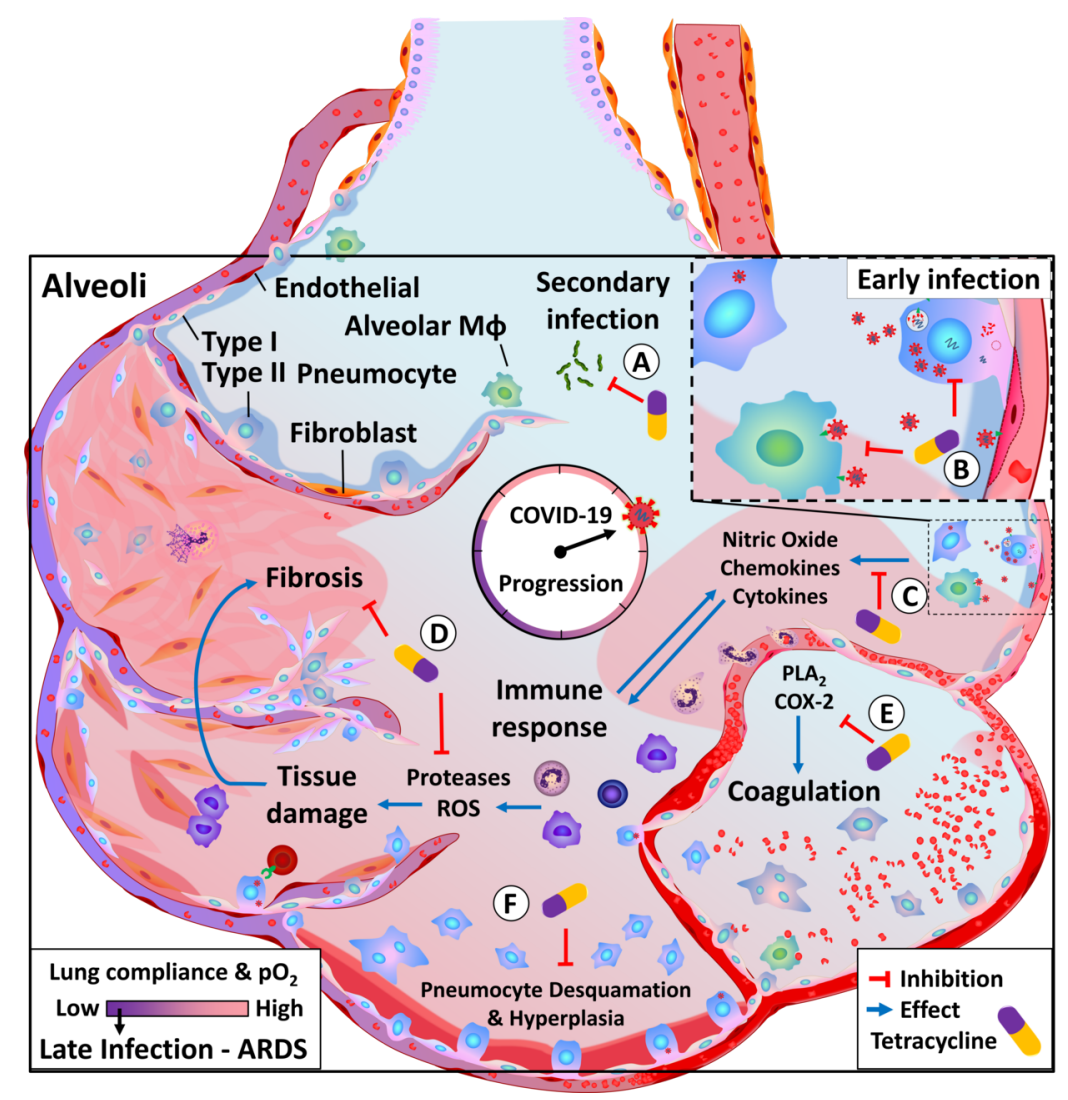


Figure 1 : COVID-19 progression and proposed pharmacological actions of immunomodulatory tetracyclines. Upon initial infection, SARS-CoV2 spreads to the lower respiratory track, infecting type II pneumocytes as well as other cell populations, such as alveolar macrophages and the endothelium. Viral infection and its cytopathic effects lead to an exacerbated immune activation, which may result in increased tissue damage and fibrosis. Alterations in lung tissue architecture (defined as Diffuse Alveolar Damage) progress into ARDS, characterised by the reduction in lung compliance and impaired blood oxygenation. Additionally, complications such as thrombosis and secondary infections are frequently observed. Tetracyclines can target this pathological process at multiple levels: A) Prevention of secondary bacterial infection. B) Reduce viral entry and replication. C) Reduce immune recruitment and activation. D) Inhibition of exacerbated inflammatory reaction via immunomodulatory effects and inhibition of oxidative stress and MMP, thus reducing tissue damage and fibrosis. E) Inhibition of enzymes involved in platelet function and coagulation. F) Inhibition of altered epithelial response.

\section{Anti-proteolitic, antioxidant and immunomodulatory activities}

Tetracycline's immunomodulatory and anti-inflammatory properties may even be of greater relevance for their protective effect against COVID-19 than their anti-infectious activity described above (Figure 1 C-E). At the time of intervention, the damaging consequences of the host response may overcome the infection itself. The lung is particularlly susceptible to the outcome of wide-spread inflammation, sequestering neutrophils and monocytes into the pulmonary tissue. In this regard, doxycycline has shown to reduce nitric oxide and chemokine production by lung epithelial cells (Hoyt et al., 2006; Raza et al., 2006), and to reduce neutrophil chemotaxis into the airspaces of the lung in vivo (Moon et al., 2012) (Figure 1C). Tetracyclines are effective in reducing the proteolytic activity derived from neutrophilic inflammation in COPD (Maisi et al., 1999), which can prevent fibrosis sequalae in ARDS survivors (Figure 1D). Of note, a retrospective multi-institutional cohort study concluded that minocycline or doxycycline treatment within a year prior to ARDS was associated with a $75 \%$ reduced likelihood for mechanical ventilation and reduced duration of mechanical ventilation and ICU stay (Byrne et al., 2020). Given the interest of tetracycline's activity for lung protection, the application of chemically modified tetracyclines (CMTs) to ARDS is not completely novel. Prophylactic CMT-3 has shown to prevent the development of ARDS in models induced by sepsis (Steinberg et al., 2005) and cardiopulmonary bypass (Carney David E. et al., 1999). Therapeutic benefit has also been achieved with CMT-3 in lung injury upon established inflammation/septic events (Roy et al., 2012; Sadowsky et al., 2015) as well as other models of lung injury. In these contexts, CMT-3 treatment was associated with a reduction in inflammation, collagen deposition and the histological lesions of ARDS (Roy et al., 2012). Mechanistically, their effects could derive from the reduction in neutrophil transmigration and neutrophil-mediated inflammation, and the direct inhibition of elastases, MMPs and oxygen radical species produced by the immune system during the inflammatory reaction, which damage alveolar-capillary basement membranes and the extracellular matrix (Figure 1D). Tetracyclines also display immunomodulatory actions in other relevant populations, such as $\mathrm{T}$ cells and macrophages. Regarding the later, in contrast to their immunosuppressive effect observed in peritoneal macrophages, it has been described that tetracyclines could potentiate the response of alveolar macrophages (Bonjoch et al., 2015). We have observed a similar effect in intestinal inflammation, where tetracyclines enhanced macrophage recruitment and response, but this resulted in accelerated differentiation into the homeostatic phenotype and improved mucosal healing (Garrido-Mesa et al., 2018). Considering that both intestinal and alveolar macrophages reside at mucosal sites and share this particular response to immunomodulatory tetracyclines, we believe that a similar protective outcome could be expected. In fact, no adverse effects have been observed upon their evaluation in ARDS. Finally, CMT-3 has also shown to prevent coagulopathy associated to ARDS, an important pathological feauture of COVID-19, which could derive from its inhibitory effects in PLA2 and COX-2, essential for platelet function (Roy et al., 2012) (Figure 1E). This synergic combination of anti-proteolitic, antioxidant and immunomodulatory activities adds to the well-known mechanisms described above, including antibiotic protection from secondary bacterial pneumonia.

In addition to these direct immunomodulatory effects, tetracyclines can also impact altered responses of the stromal compartment (Figure 1F). On the basis that ACE2 inhibition due to viral entry could contribute 
to lung injury in COVID-19, a recent study has shown that CMT-3 is a great candidate to reverse the altered gene expression pattern of lung cells upon ACE2 inhibition (He and Garmire, 2020). Finally, for a complete understanding of the mechanisms behind the effects observed for tetracyclines in ARDS and their potential for COVID-19, it is also worth mentioning their ability to concentrate at sites of inflammation and tissue injury, which might potentiate tetracycline's pharmacological effects. This is explained by the increased tetracycline uptake observed with increasing temperature, as well as in specific cell types, such as neutrophils and alveolar macrophages, where tigecycline can be found up to 78-times more concentrated than in blood.

\section{Clinical evidence}

Overall, whilst tetracycline's direct anti-viral activity against SARS-CoV2 is not yet strongly supported by experimental data, their above described beneficial mechanisms in ARDS can be directly translated to COVID-19-induced ARDS. Furthermore, our hypothesis of the therapeutic potential of repurposing tetracyclines for COVID-19 treatment is getting the support of the clinical reports published up to date. These are scarce, but positive and promising. For example, rapid clinical improvement was reported in 4 high risk COVID-19 patients after doxycycline treatment (Yates et al., 2020). A multicenter prospective observational study including 38 COVID-19 patients treated with tetracyclines reported a remarkable resolution of mild symptoms within the first week of treatment (Gironi et al., 2020). In a larger study, early treatment with doxycycline in 89 high-risk COVID-19 patients was associated with early clinical recovery, decreased hospitalization and decreased mortality (Alam et al., 2020). Furthermore, doxycycline has been used for COVID-19 treatment in combination with hydroxychloroquine or lopinavir, reporting an overall $4.2 \%$ fatality rate vs $27 \%$ and $23 \%$ for monotherapy, respectively (Cag et al., 2021). In another study, in combination with ivermectin, doxycycline helped to reduce disease progression and the time to recovery in patients with severe COVID-19. The mortality rate was also reduced from $22.72 \%$ to $0 \%$ compared to standard care with azithromycin (Hashim et al., 2020). Altogether, we believe that these reports provide sufficient clinical evidence to support the inclusion of tetracyclines in ARDS and COVID-19 therapeutic management.

\section{Conclusion}

From a global perspective, the safe application of therapeutic strategies to COVID-19 can only be effectively achieved by drug repurposing. In this regard, tetracyclines combine potentially beneficial direct anti-viral activity with anti-inflammatory and immunomodulatory properies, as well as anti-bacterial effect, that can together assist in the therapeutic management of severe cases of COVID-19. Their confirmed benefit in the treatment of ARDS strongly supports the notion that they would be of interest for the treatment COVID19. In addition, Tetracyclines are safe, well-knownand economically accessible, with doxycycline among the WHO essential medicines. This positions them as excellent therapeutic candidates for the management of COVID-19, particularly in developing countries where successful vaccination programs have yet to be established and access to other therapeutic resources is scarce.

\section{REFERENCES}

Alam, M.M., Mahmud, S., Rahman, M.M., Simpson, J., Aggarwal, S., and Ahmed, Z. (2020). Clinical Outcomes of Early Treatment With Doxycycline for 89 High-Risk COVID-19 Patients in Long-Term Care Facilities in New York. Cureus 12 : e9658.

Bharadwaj, S., Lee, K.E., Dwivedi, V.D., and Kang, S.G. (2020). Computational insights into tetracyclines as inhibitors against SARS-CoV-2 Mpro via combinatorial molecular simulation calculations. Life Sci. 257 : 118080 .

Bonjoch, L., Gea-Sorlí, S., Jordan, J., and Closa, D. (2015). Minocycline inhibits peritoneal macrophages but activates alveolar macrophages in acute pancreatitis. J. Physiol. Biochem. 71 : 839-846. 
Byrne, J., Shakur, R., Collins, J., Becker, S., Young, C., Boyce, H., et al. (2020). Prophylaxis with tetracyclines in ARDS: Potential therapy for COVID-19-induced ARDS? | medRxiv.

Cag, Y., Icten, S., Isik-Goren, B., Baysal, N.B., Bektas, B., Selvi, E., et al. (2021). A novel approach to managing COVID-19 patients; results of lopinavir plus doxycycline cohort. Eur. J. Clin. Microbiol. Infect. Dis. Off. Publ. Eur. Soc. Clin. Microbiol. 40 : 407-411.

Carney David E., Lutz Charles J., Picone Anthony L., Gatto Louis A., Ramamurthy N. S., Golub Lorne M., et al. (1999). Matrix Metalloproteinase Inhibitor Prevents Acute Lung Injury After Cardiopulmonary Bypass. Circulation $100:$ 400-406.

Dutta, K., and Basu, A. (2011). Use of minocycline in viral infections. Indian J. Med. Res. 133 : 467-470.

Garrido-Mesa, J., Rodríguez-Nogales, A., Algieri, F., Vezza, T., Hidalgo-Garcia, L., Garrido-Barros, M., et al. (2018). Immunomodulatory tetracyclines shape the intestinal inflammatory response inducing mucosal healing and resolution. Br. J. Pharmacol.

Gendrot, M., Andreani, J., Jardot, P., Hutter, S., Delandre, O., Boxberger, M., et al. (2020). In Vitro Antiviral Activity of Doxycycline against SARS-CoV-2. Mol. Basel Switz. 25 :.

Gironi, L.C., Damiani, G., Zavattaro, E., Pacifico, A., Santus, P., Pigatto, P.D.M., et al. (2020). Tetracyclines in COVID-19 patients quarantined at home: Literature evidence supporting real-world data from a multicenter observational study targeting inflammatory and infectious dermatoses. Dermatol. Ther. e14694.

Hashim, H.A., Maulood, M.F., Rasheed, A.M., Fatak, D.F., Kabah, K.K., and Abdulamir, A.S. (2020). Controlled randomized clinical trial on using Ivermectin with Doxycycline for treating COVID-19 patients in Baghdad, Iraq (Infectious Diseases (except HIV/AIDS)).

He, B., and Garmire, L. (2020). Prediction of repurposed drugs for treating lung injury in COVID-19. F1000Research $9: 609$.

Hoyt, J.C., Ballering, J., Numanami, H., Hayden, J.M., and Robbins, R.A. (2006). Doxycycline modulates nitric oxide production in murine lung epithelial cells. J. Immunol. Baltim. Md 1950176 : 567-572.

Maisi, P., Kiili, M., Raulo, S.M., Pirilä, E., and Sorsa, T. (1999). MMP Inhibition by Chemically Modified Tetracycline-3 (CMT-3) in Equine Pulmonary Epithelial Lining Fluid. Ann. N. Y. Acad. Sci. 878 : 675-677.

Moon, A., Gil, S., Gill, S.E., Chen, P., and Matute-Bello, G. (2012). Doxycycline impairs neutrophil migration to the airspaces of the lung in mice exposed to intratracheal lipopolysaccharide. J. Inflamm. Lond. Engl. 9 $: 31$.

Raza, M., Ballering, J.G., Hayden, J.M., Robbins, R.A., and Hoyt, J.C. (2006). Doxycycline decreases monocyte chemoattractant protein-1 in human lung epithelial cells. Exp. Lung Res. 32 : 15-26.

Roy, S.K., Kubiak, B.D., Albert, S.P., Vieau, C.J., Gatto, L., Golub, L., et al. (2012). Chemically Modified Tetracycline 3 Prevents Acute Respiratory Distress Syndrome in a Porcine Model of Sepsis + Ischemia/Reperfusion-Induced Lung Injury. Shock 37 : 424-432.

Sachdeva, C., Wadhwa, A., Kumari, A., Hussain, F., Jha, P., and Kaushik, N.K. (2020). In silico Potential of Approved Antimalarial Drugs for Repurposing Against COVID-19. Omics J. Integr. Biol. 24 : 568-580.

Sadowsky, D., Nieman, G., Barclay, D., Mi, Q., Zamora, R., Constantine, G., et al. (2015). Impact of chemically-modified tetracycline 3 on intertwined physiological, biochemical, and inflammatory networks in porcine sepsis/ARDS. Int. J. Burns Trauma 5 : 22-35.

Sayed, A.M., Khalaf, A.M., Abdelrahim, M.E.A., and Elgendy, M.O. (2020). Repurposing of some antiinfective drugs for COVID-19 treatment: A surveillance study supported by an in silico investigation. Int. J. Clin. Pract. e13877. 
Steinberg, J., Halter, J., Schiller, H., Gatto, L., Carney, D., Lee, H.-M., et al. (2005). Chemically modified tetracycline prevents the development of septic shock and acute respiratory distress syndrome in a clinically applicable porcine model. Shock Augusta Ga 24 : 348-356.

Szeto, G.L., Brice, A.K., Yang, H.-C., Barber, S.A., Siliciano, R.F., and Clements, J.E. (2010). Minocycline attenuates HIV infection and reactivation by suppressing cellular activation in human CD4+ T cells. J. Infect. Dis. 201 : 1132-1140.

Wu, C., Liu, Y., Yang, Y., Zhang, P., Zhong, W., Wang, Y., et al. (2020). Analysis of therapeutic targets for SARS-CoV-2 and discovery of potential drugs by computational methods. Acta Pharm. Sin. B $10: 766-788$.

Yates, P.A., Newman, S.A., Oshry, L.J., Glassman, R.H., Leone, A.M., and Reichel, E. (2020). Doxycycline treatment of high-risk COVID-19-positive patients with comorbid pulmonary disease. Ther. Adv. Respir. Dis.14 : 1753466620951053.

Zhao, T.Y., and Patankar, N.A. (2021). Tetracycline as an inhibitor to the coronavirus SARS-CoV-2. J. Cell. Biochem. jcb.29909. 